\title{
Morfometría de los equinodeos de la Familia Cassidulidae (Echinoidea: Cassiduloida)
}

\author{
Alejandra Martínez-Meloํㅡㄹ Efraín De Luna ${ }^{2}$ \& Blanca Estela Buitrón-Sánchez ${ }^{1}$ \\ 1. Departamento de Paleontología, Instituto de Geología, Universidad Nacional Autónoma de México Ciudad \\ Universitaria, Delegación Coyoacán CDMX 04510, México; a.mtz.melo@gmail.com, blancab@unam.mx \\ 2. Instituto de Ecología, INECOL. Carretera Antigua a Coatepec No. 351, Col. El Haya. C.P. 91070, Xalapa, Veracruz, \\ México; efrain.deluna@inecol.mx
}

Recibido 16-XII-2016. Corregido 07-I-2017. Aceptado 02-V-2017.

\begin{abstract}
Morphometrics of echinoids in the Family Cassidulidae (Echinoidea: Cassiduloida). Cassidulidae is the type family of the order Cassiduloida, and contains five genera whose morphology has complicated their taxonomic study: Australanthus, Cassidulus, Eurhodia, Paralampas, and Rhyncholampas. Many authors have applied traditional morphometric analysis (mainly length, width, and height) with varying success. We present the first approach with geometric morphometrics (three outlines: aboral, left, and posterior). Principal component analyses on aligned semi-landmarks detect shape deformations among the five genera. For future studies, we recommend larger samples, and the inclusion of size and other characters such as the ambulacra and peristome. Rev. Biol. Trop. 65(Suppl. 1): S233-S243. Epub 2017 November 01.
\end{abstract}

Key words: Geometric Morphometrics; Taxonomy; semi-landmarks; Principal Component Analysis.

El orden Cassiduloida L. Agassiz \& Desor, 1847 es considerado como un grupo paraflético (Kier, 1962). Cuenta con 67 géneros y aproximadamente 800 especies descritas, de las cuales la mayoría son fósiles (Mooi, 1990). La familia Cassidulidae L. Agassiz \& Desor, 1847 data del Maestrichtiano (Cretácico Tardío) y presentó su mayor diversidad específica durante el Eoceno. Actualmente es una de las dos familias de casiduloides con representantes actuales.

El trabajo más importante acerca de la clasificación de los equinoideos, hasta la fecha, es el que realizó Mortensen (1948), donde mencionó que la familia Cassidulidae contiene 26 géneros. Kier (1962) publicó una extensa revisión sobre la morfología y clasificación de los taxa pertenecientes al orden Cassiduloida, incluyendo dentro de la familia Cassidulidae los géneros Rhyncholampas A. Agassiz, 1869, Cassidulus Lamarck, 1801, Rhyncho- pygus d'Orbigny, 1856 (con sus sinonimias, Paralampas Duncan \& Salden, 1882 y Procassidulus Lambert, 1918); Nucleopygus L. Agassiz, 1840 (con sus sinónimos Lychnidius Pomel, 1883 y Porobrissus Lambert, 1916), Hypsopygaster Bajarunas, 1915 y Ochetes Pomel, 1883.

Mooi (1990) enlistó 30 especies recientes del orden Cassiduloida; en esta propuesta incluyó a cinco géneros y ocho especies de casidúlidos: Cassidulus caribaearum Lamarck, 1801, C. infidus Mortensen, 1948, C. mitis Krau, 1954, C. malayanus (Mortensen, 1948), Eurhodia relicta Mooi, 1990, Oligopodia epigonus (van Maartens, 1865), Rhyncholampas pacificus (A. Agassiz, 1863) y Studeria recens (A. Agassiz, 1863). 
Suter (1994a) trabajó con la morfología de casiduloides (sensu Kier), analizando 40 caracteres morfológicos de 97 especies fósiles y recientes para proponer sus relaciones filogenéticas. Sus resultados sugieren el reacomodo de distintos géneros; el cladograma final requiere algunos cambios que él mismo indicó, con la finalidad de proponer las tendencias evolutivas de varios caracteres. También sugirió el uso de métodos moleculares para apoyar o desmentir la relación; mencionó que los trabajos que se habían realizado a la fecha no contemplaban los suficientes caracteres que soportaran una filogenia monofilética, incluyendo muchas veces caracteres presentes en otros grupos como en los clypeasteroides; es por ello que Suter (1994b) intentó proponer la monofilia del taxón, utilizando 71 caracteres morfológicos de las 30 especies actuales que cita Mooi (1990). Sin embargo, sus resultados no soportan la monofilia del taxón.

Por último, Suter (1994c) presenta una propuesta de filogenia para los casiduloides, basándose en Kier (Ibid.) pero incluyó algunos elementos de los órdenes Clypeasteroida L. Agassiz, 1835, Oligopygoida Kier, 1867, Neolampadoida Philip, 1963 y de la familia Conoclypidae Zittel, 1879.

Wilkinson, Suter y Shires (1996) aplicaron el método Cladista del Consenso Reducido (RCC por sus siglas en inglés) a los datos de Suter (1994a y 1994b) para resolver mejor las relaciones filogenéticas de los casiduloides. Como resultado obtuvieron un cladograma, prácticamente no resuelto en el cual se separan cuatro pequeños clados; en particular, el clado quien comprende los géneros Oligopodia, Rhynchopygus, Eurhodia, Rhyncholampas y Cassidulus. Smith y Kroh (2011) consideraron a la familia Cassidulidae con cinco géneros: Cassidulus, Eurhodia, Glossaster Lambert, 1918, Paralampas y Rhyncholampas.

Aun existiendo estudios taxonómicos sólidos sobre la familia Cassidulidae, todavía hay muchas dudas con respecto a su diagnosis que no permiten establecer exactamente el número de géneros, y sus respectivas especies, que la integran. Los únicos géneros descritos para la familia Cassidulidae y que siempre han permanecido en ella son Cassidulus y Rhyncholampas.

El género Cassidulus Lamarck, 1801 tiene cuatro especies recientes (Cassidulus caribaearum Lamarck, 1801, C. infidus Mortensen, 1948, C. mitis Krau, 1954 y C. malayanus Mortensen, 1948), mientras que el género Rhyncholampas A. Agassiz, 1863 conserva sólo una especie reciente, Rhyncholampas pacificus (A. Agassiz, 1863). Cuando Lamarck (1801) propuso al género Cassidulus, le asignó tres especies, Cassidulus caribaearum, C. scutellanus y $C$. belgicus, designando a la primera como especie tipo.

Agassiz y Desor (1847) designaron a Cassidulus marmini como especie tipo del género Rhynchopygus. Agassiz (1863) describió la especie Pygorhynchus pacificus, siendo P. lapis cancri la especie tipo del género (Mortensen, 1948).

Agassiz (1869) señala que el género Cassidulus (sensu Lamarck, 1801), contiene dos especies tipo: Cassidulus lapis cancri y $C$. caribaearum, cuando en realidad Lamarck establece a ésta última como especie tipo y nunca menciona a $C$. lapis cancri en su trabajo. De cualquier manera, Agassiz toma la decisión de crear el género Rhyncholampas como una respuesta a las diferencias taxonómicas con el género Cassidulus y señala: "propongo retener temporalmente al género Cassidulus para las especies fósiles relacionadas con $\mathrm{C}$. lapis cancri y dejar a los paleontólogos la tarea de delimitar adecuadamente dicho género y separar del género Cassidulus, bajo el nombre de Rhyncholampas a un nuevo género que incluya $a$ Cassidulus caribaearum y a su representante de la Costa Occidental, el cual fue llamado originalmente Pygorhynchus pacificus".

Algunos autores han utilizado los análisis de morfometría tradicional para resolver diferentes problemas taxonómicos intra e intergenéricos. Sobre los análisis morfométricos aplicados a casiduloides existen los trabajos de Kier (1967), quien revisó morfológica y 
morfométricamente a las especies del género Oligopygus tomando en cuenta diferentes caracteres de la testa, así como el número de poros en los ambulacros.

Carter y Beisel (1987) transfirieron a Cassidulus trojanus Cooke, 1942 al género Eurhodia. Se basaron en la comparación intergenérica de la relación ancho:largo de la testa utilizando especies de los géneros Cassidulus, Eurhodia y Rhyncholampas.

Zachos y Molineux (2003) publicaron un listado de especies fósiles del Eoceno de Texas, EUA y compararon cuatro especies del género Fibularia Lamarck, 1816. Graficaron tallas de la testa como lo propuso Kier (1967), pero comparando el largo de la testa contra la redondez de Fibularia texana (Twitchell, 1915).

Ciampaglio y D’Orazio (2007) realizaron el análisis morfométrico de las especies Echinolampas appendiculata Emmons, 1858, Rhyncholampas carolinensis (Twitchell, 1915) y Eurhodia rugosa (Ravenel, 1848) para conocer las relaciones heterocrónicas entre ellas y demostraron que hay una gran relación entre los géneros Eurhodia y Rhyncholampas.

Martínez-Melo (2008) realizó un análisis morfométrico que muestra una posible relación heterocrónica entre las especies recientes Cassidulus caribaearum y Rhyncholampas pacificus, distribuidas en las costas mexicanas. Al parecer la más notable diferencia morfológica entre estas especies, es el número de placas que forman el plastrón, pues en los primeros estadios es el mismo número, pero en el estadio adulto es mayor para el género Rhyncholampas.

Las técnicas de la morfometría tradicional, basadas en la aplicación de métodos estadísticos multivariados sobre un conjunto de variables lineales, mientras que la morfometría geométrica es un conjunto de nuevas técnicas apropiadas para el estudio estadístico de la variación y covariación de la forma de una estructura, concebida ésta como los atributos de una configuración de coordenadas que resultan invariantes con respecto a los efectos de traslación, rotación y escala geométrica (Rohlf, 2000).

Los trabajos de morfometría antes mencionados prueban que ésta es una herramienta que ayuda a resolver problemas taxonómicos, sobre todo a nivel intra e intergenérico. Tomando en cuenta que, mediante la morfometría geométrica se puede analizar las formas de las estructuras y no sólo sus tallas, en este trabajo se propone el uso de este tipo de análisis para reconocer las zonas de mayor variación morfológica de las testas de la familia Cassidulidae. Como un primer acercamiento se tomaron en cuenta tres parámetros de la testa, el aboral, lateral y posterior de representantes de la familia Cassidulidae.

\section{MATERIAL Y MÉTODO}

Se analizaron tres ejemplares de cada uno de los cinco géneros (Cuadro 1) incluidos en la Familia Cassidulidae (sensu Kier, 1962) y depositados en tres colecciones: 1) USNM (National Museum of Natural History, Smithsonian Institution, Estados Unidos de América), 2) ICML-UNAM (Colección Nacional de Equinodermos "Ma. Elena Caso Muñoz", Instituto de Ciencias del Mar y Limnología, México) y 3) CAS-IZ (Invertebrate Zoology Collection, California Academy of Sciences, Estados Unidos de América). Debido al escaso registro del género Paralampas, fue necesario completar su registro fotográfico utilizando los recursos en línea del Muséum National d'Histoire Naturelle, Francia (MNHN, 2016), así como las imágenes publicadas por Duncan y Sladen (1882).

Se tomaron tres fotografías (aboral, lateral izquierda y posterior), con una cámara Sony $\alpha 37$, para cada ejemplar incluido en el análisis. Se registraron solamente semimarcas, debido a que se trataba de contornos sin puntos 
CUADRO 1

Ejemplares de la familia Cassidulidae incluidos en el análisis de morfometría geométrica

TABLE 1

Specimens of family Cassidulidae included in the analysis of geometric morphometrics

\begin{tabular}{lll} 
& \multicolumn{1}{c}{ Especie } & \multicolumn{1}{c}{ Núm. Catálogo } \\
1 & Australanthus florescens (Gregory, 1892) & USNM 460547 \\
2 & Australanthus florescens (Gregory, 1892) & USNM 460547 \\
3 & Australanthus longianus (Gregory, 1890) & USNM 460548 \\
4 & Cassidulus caribaearum Lamarck, 1801 & ICML-UNAM 4.96.18 \\
5 & Cassidulus mitis Krau, 1954 & CAS-IZ 116110 \\
6 & Cassidulus trojanus Cooke, 1942 & USNM 498996 \\
7 & Eurhodia baumi Kier, 1980 & USNM 264043 \\
8 & Eurhodia holmesi Twitchell, 1915 & USNM 264592 \\
9 & Eurhodia rugosa Ravenel, 1848 & USNM 562300 \\
10 & Paralampas pileus Duncan \& Sladen, 1882 & UCMP C3637 \\
11 & Paralampas pileus Duncan \& Sladen, 1882 & Duncan \& Sladen, 1882 \\
12 & Paralampas rancureli Tessier \& Roman, 1973 & MNHN.F.R06427 \\
13 & Rhyncholampas evergladensis (Mansfield, 1932) & USNM 648146 \\
14 & Rhyncholampas gouldii (Bouvé, 1846) & MCZ \\
15 & Rhyncholampas pacificus (A. Agassiz, 1863) & ICML-UNAM 4.48.4 \\
\hline
\end{tabular}

anató- micos discretos; con el fin de colocar las semi- marcas de manera equidistante sobre los tres contornos de la testa, se trazaron lineas guías con el programa Makefan8 (Sheets, 2016b). Se construyeron archivos TPS con el programa TPSutil ver. 1.69 (Rohlf, 2016b)

Se digitalizaron coordenadas Cartesianas de los puntos en el programa TPSdig2 ver 2.26 (Rohlf, 2016a) (Fig. 1). En el contorno de la superficie aboral se construyó un círculo con 24 radios y se colocaron los puntos en donde los radios tocan el margen (Fig. $1 \mathrm{~A}$ ); para describir las curvas del contorno lateral de la testa se construyó un peine de 30 líneas equidistantes (Fig. 1 B) y en el contorno de la vista posterior se construyó un círculo de 40 radios (Fig. 1 C).

Se utilizó la herramienta SemiLand8 en CoordGen8 (Sheets, 2016a) para alinear las coordenadas Procrustes (Fig. 2). Los tres protocolos de alineamiento se definieron como contornos cerrados. Las coordenadas superpuestas $\mathrm{y}$ alineadas en los quince especimenes se les sometió a un análisis de componentes principales (PCA) en PCAGen8 (Sheets, 2001). Se obtuvieron los autovalores de los componentes principales y el porcentaje de varianza que aporta cada componente así como las gradillas de deformación con base en el componente principal 1 (Fig. 3) y la gráfica de los dos primeros componentes principales para los tres contornos (Figs. 4, 5, 6).

\section{RESULTADOS}

Se digitalizaron 24 semimarcas para el contorno aboral, 30 para el contorno lateral izquierdo y 26 para el contorno posterior, suficientes para la completa descripción de los contornos. Estos datos de cada configuración de puntos se sometieron a la superposición de Procrustes y alineamiento de semimarcas (Fig. 2). 

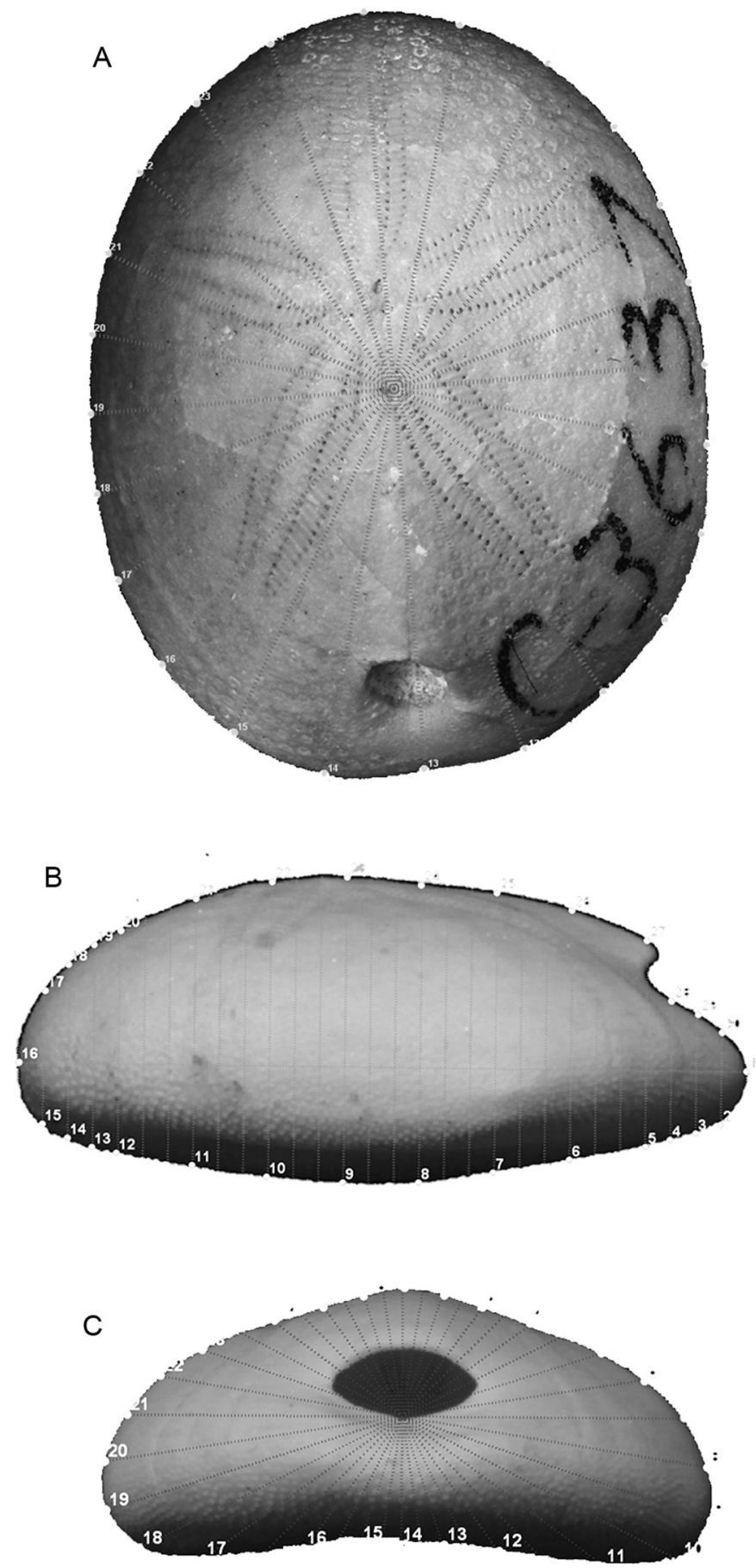

Fig. 1. Peines construidos en SemiLand8 para la digitalización de las semimarcas. Fig. 1. Fans built in SemiLand8 for the digitalization of semilandmarks. 

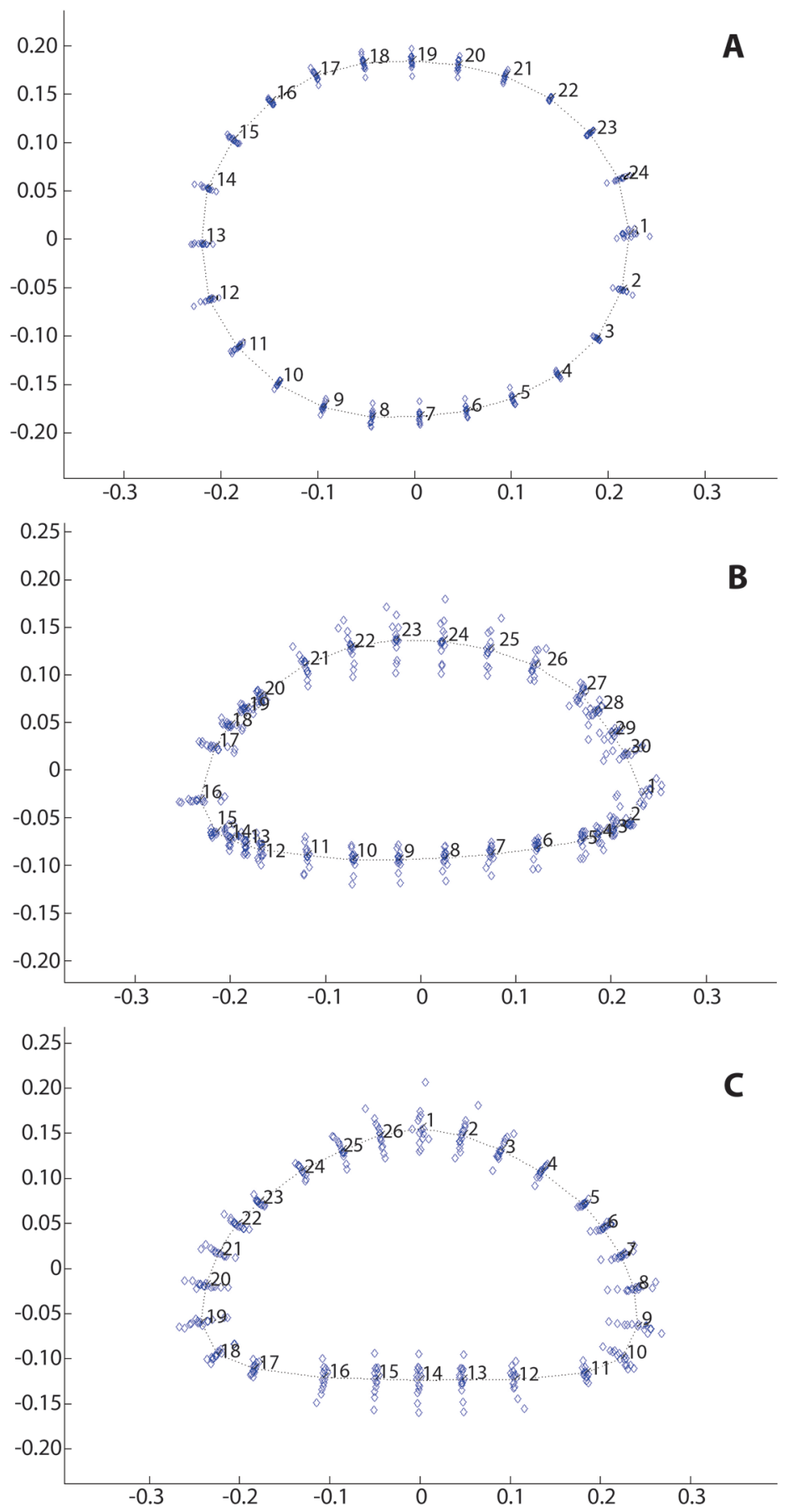

Fig. 2. Configuraciones de semimmarcas superpuestas (Procrustes) y alineadas. A, contorno aboral; $\mathrm{B}$, contorno lateral; $\mathrm{C}$, contorno posterior.

Fig. 2. Procrustes superimposition. A. Aboral outline; B. lateral outline; C. posterior outline. 


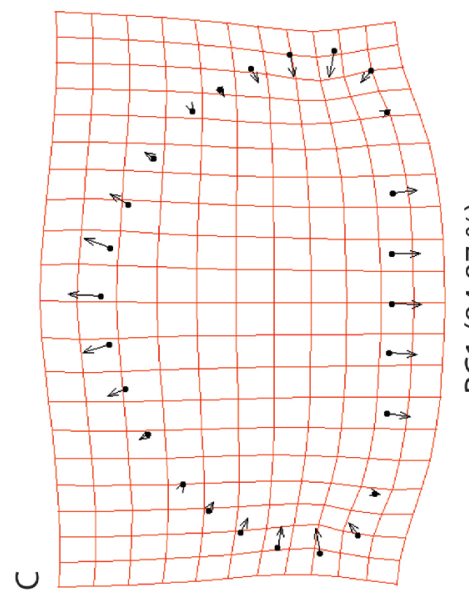

Se obtuvieron los componentes principales para cada uno de los tres contornos (vistas aboral, lateral y posterior). El primer componente principal (PC1) explica porcentajes de varianza alrededor del $80 \%$.

Las gradillas de deformación del CP1 muestran la dirección y magnitud de los vectores de deformación de cada una de las semimarcas. En vista aboral (Fig. 3 A) el cambio es entre formas redondeadas y formas más estrechas y alargadas. La vista lateral (Fig. 3 B) cambia en la zona del ápice y la testa. La vista posterior (Fig. $3 \mathrm{C}$ ) cambia en la región de la testa.

\section{DISCUSIÓN}

El número de especímenes por género es obviamente muy reducido para hacer cualquier tipo de inferencias estadísticas. Por ahora este trabajo demuestra el poder descriptivo de las semimarcas para analizar cuantitativamente los contornos en las tres vistas. Un mayor número de ejemplares permitiría examinar con análisis de disparidad si una vista es mas variable que otra. También podría examinarse si un género es más variable que otro.

Por el momento los PCA solo permiten postular que estas tres vistas podrían ser un caracter informativo para discriminar géneros.

$\infty$

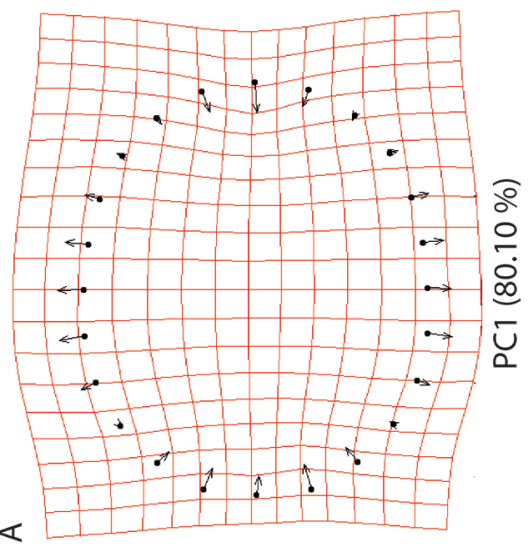

Esto concuerda con lo mencionado por Kier (1967), quien menciona que el alto de la testa es menos significativo que el ancho de la misma para caracterizar a las especies del género Oligopygus y distinguirlas del género Haimea.

El género Australanthus es ligeramente más variable en el contorno lateral (Fig. 5); esto puede deberse a que $A$. longianus tiene un ápice más alto que $A$. florescens, pero sus contornos aboral y posterior son muy similares. El género Cassidulus es poco variable en su contorno aboral (Fig. 4), pero presenta una mayor variabilidad en la vista lateral (Fig. 5).

En los tres PCA se detectó la variación dentro del género Eurhodia (Fig. 4, 5, 6), esto debido principalmente a la inclusión de $E$. rugosa, especie con un endoesqueleto de forma elíptica, muy alargado y aplanado; por otro 


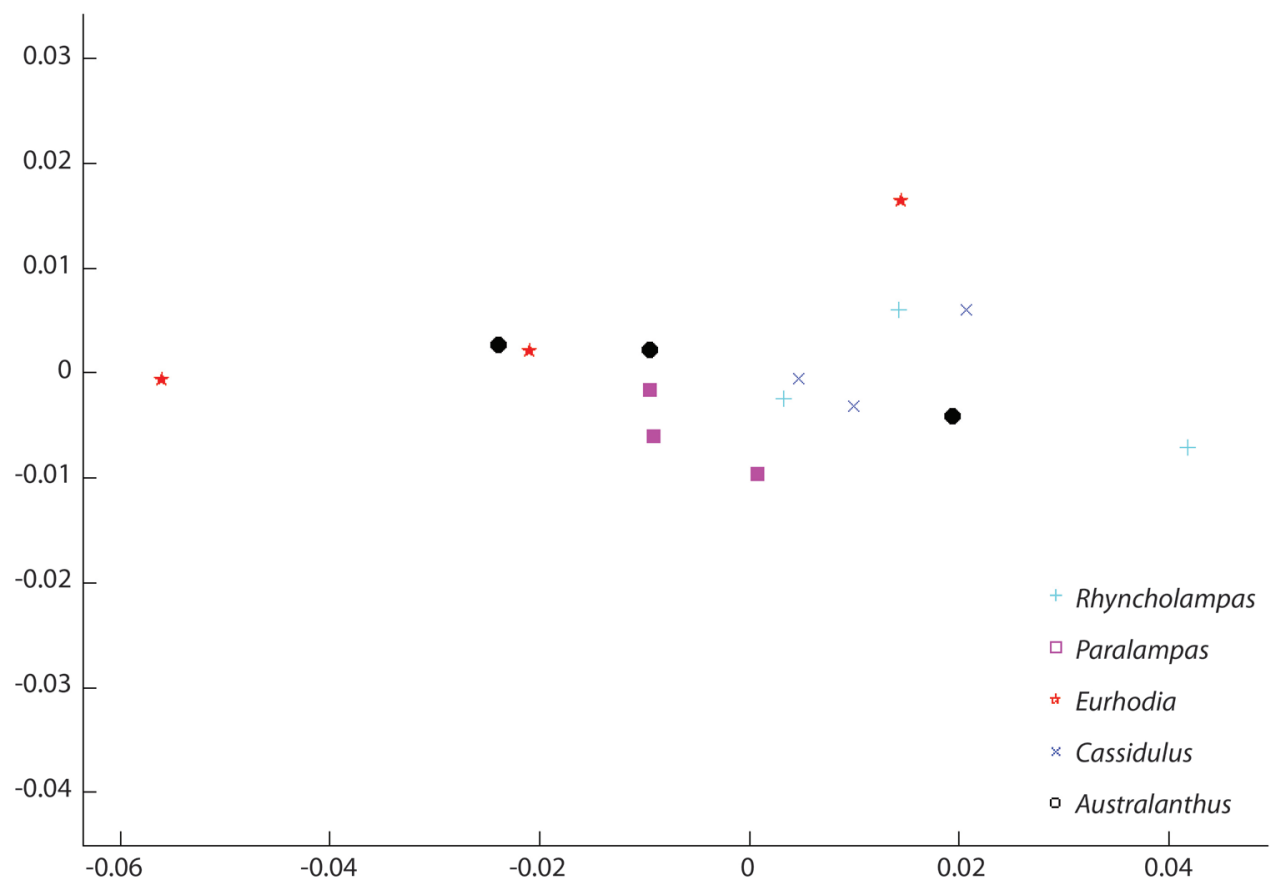

Fig. 4. Gráficas de componentes principales 1 y 2 del contorno aboral.

Fig. 4. Principal components 1 and 2 of aboral outline.

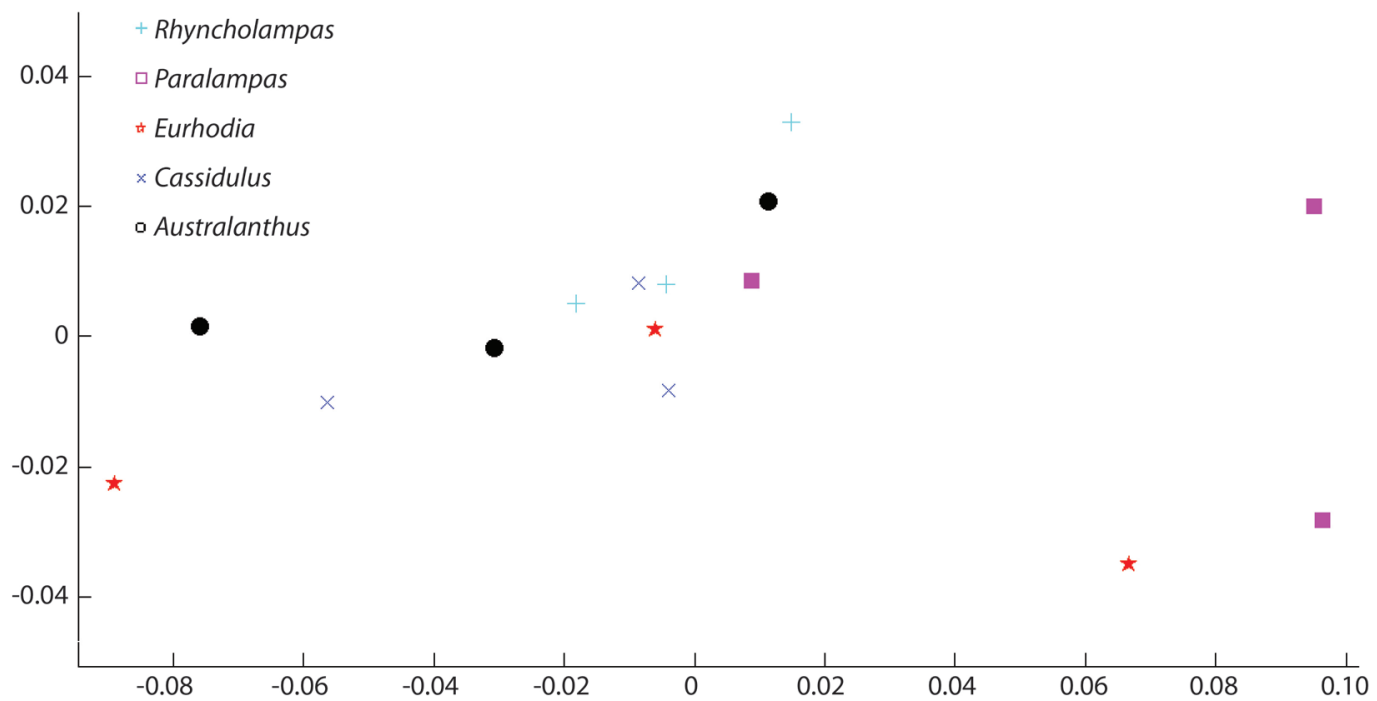

Fig. 5. Gráficas de componentes principales 1 y 2 de la vista lateral.

Fig. 5. Principal components 1 and 2 of lateral outline. 


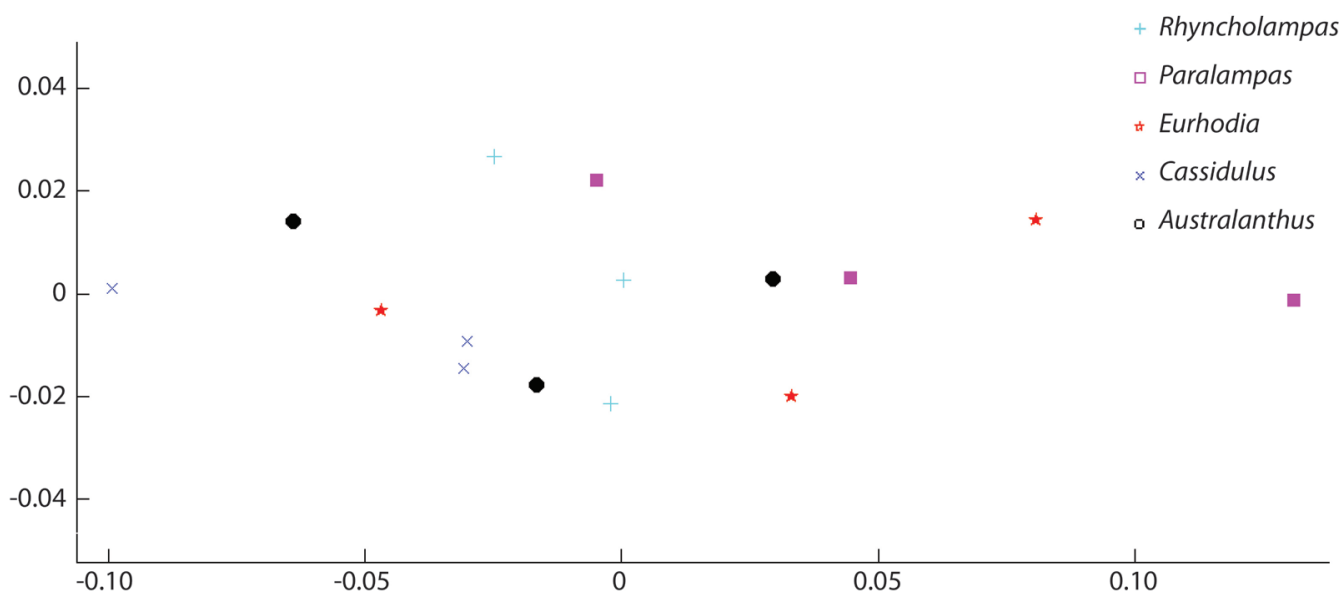

Fig. 6. Gráficas de componentes principales 1 y 2 de la vista posterior.

Fig. 6. Principal components 1 and 2 of posterior outline.

lado, E. holmesi es una especie de testa con forma semicircular con una ligera prolongación en el periprocto.

El uso de imágenes de bibliografía del género Paralampas fue necesario debido al difícil acceso a ejemplares. Ésta práctica es correcta debido a que se utilizaron referencias formales, sin embargo, es necesario ampliar el registro fotográfico de ejemplares albergados en colecciones, pues se observan diferencias entre el ejemplar de bibliografía y el fotografiado, pero esta diferencia no es anomala en el contexto de las graficas del PC1 y PC2.

El género Rhyncholampas muestra una mayor variación en el contorno aboral, esto debido a que $R$. gouldii es de ámbito circular, $R$. evergladensis se ensancha en el tercio posterior y $R$. pacificus presenta una ligera proyección con relación a la presencia del periprocto. Los tres contornos de los géneros Australanthus, Cassidulus y Rhyncholampas son menos variables, sin embargo, sus distribuciones se sobrelapan en los tres análisis.

Este primer ensayo muestra el potencial de la aplicación de semimarcas para la descripción cuantitativa de la forma de los equinoideos; las coordenadas podrían analizarse filogenéticamente para delimitar taxonómicamente a los géneros o las especies de la familia Cassidulidae; los análisis morfométricos de los contornos nos dan una primera idea de los caracteres a los que debemos poner especial atención, además de sentar las bases para posteriores análisis taxonómicos. Es necesario profundizar en el análisis de las testas de los géneros de la familia Cassidulidae incluyendo más ejemplares y utilizando marcas y semimarcas que describan caracteres cualitativos, como las curvas de los pares de poros que forman las ambulacros petaloides o la curva del contorno del peristoma. Al contar con dicha información será posible tener análisis morfométricos y filogenéticos robustos que colaboren a la correcta clasificación de los géneros y las especies de la familia Cassidulidae.

\section{AGRADECIMIENTOS}

Se agradece a Kathy Hollis y Dan Levin, del Departamento de Paleontología del Smithsonian Museum of Natural History 
(Wash- ington D.C., E.U.A.) a Jessica Cundiff, del Departamento de Paleontología del Museum of Comparative Zoology, University of Harvard (Boston, Massachussetts, E.U.A.) y al perso- nal del University of California, Museum of Paleontology (Berkeley, California, E.U.A.) por su colaboración para obtener las imágenes de los ejemplares fotografiados. Esta investi- gación fue financiada por el proyecto UNAM- DGAPA-PAPIIT IN108717.

\section{RESUMEN}

Cassidulidae es la familia tipo del orden Cassi- duloida y contiene cinco géneros cuya morfología ha complicado su estudio taxonómico: Australanthus, Cassidulus, Eurhodia, Paralampas, y Rhyncholampas. Varios autores han aplicado análisis morfométricos tradicionales (principalmente largo, ancho y alto) con éxito variable. Nosotros presentamos el primer enfoque con morfometría geométrica (tres contornos: aboral, izquierdo, y posterior). Los análisis de componentes principales de las coordenadas alineadas de semimarcas detectan deformaciones entre los cinco géneros. Para estudios futuros, nosotros recomendamos muestras más grandes, y la inclusión de la talla y caracteres cualitativos como el ambulacro y el peristomo.

Palabras clave: Morfometría geométrica; Taxonomía; semimarcas; Análisis de Componentes Principales.

\section{REFERENCIAS}

Agassiz, A. (1863). List of the echinoderms sent to different institutions in Exchange for other specimens, with annotations. Bulletin Of The Museum Of Comparative Zoology, 1 (2), 17-28.

Agassiz, A. (1869). Preliminary report on the echini and starfishes dredged in deep water between Cuba And The Florida Reef. Por L. F. De Pourtalès. Bulletin Of The Museum Of Comparative Zoölogy, 1 (9), 253-308.

Agassiz, L., \& Desor, E. (1847). Catalogue raisonné des espèces, de genres, et des familles d'echinides. Parte 1. En Annales des Sciences Naturelles, 3 (7), 129-168.
Carter, B. D., \& Beisel, T. H. (1987). “Cassidulus" trojanus belongs in the genus Eurhodia (Echinoidea) based upon new criteria. The paleontological society. Journal of Paleontology, 61, 5.

Ciampaglio, N. Ch., \& D’Orazio, A. E. (2007). Heterochrony within the cassiduloid echinoids from the Castle Hayne limestone of southeastern North Carolina. Historical Biology, 19 (4), 301-313.

Duncan, P. M., \& Sladen, W. P. (1882). The fossil Echinoidea from the Ranikiot Series of Nummulitic strata in western Sind. Palaeontologica Indica, 14th series, 1 (3), 21-100, pl. 5-20.

Kier, P. M. (1962). Revision of the cassiduloid echinoids. Smithsonian Miscellaneus Collections, 144 (3), 1-262.

Kier, P. M. (1967). Revision Of The Oligopygoid Echinoids. Smithsonian Miscellaneous Collections, 152 (2), 1-149.

Lamarck, J. B. (1801). Systême des animaux sans vertèbres. 1-349.

Martínez-Melo, A. (2008). Relación heterocrónica entre Rhyncholampas pacificus (A. Agassiz, 1863) y Cassidulus caribaearum Lamarck, 1801. Posgrado de Ciencias del Mar y Limnología, Universidad Nacional Autónoma de México, México.

MNHN. (2016). https://science.mnhn.fr/taxon/species/ paralampas/rancureli

Mooi, R. (1990). Living cassiduloids (Echinodermata: Echinoidea): A key and annotated list. Proceedings of the Biological Society of Washington, 103, 63-85.

Mortensen, T. (1948). A monograph of the Echinoidea. IV. 1. Holectypoida, Cassiduloida. C. A. Rietzel, Copenhagen, Dinamarca.

Rohlf, F. J. (2000). Statistical power comparisons among alternative morphometric methods. American Journal of Physical Anthropology, 111, 463-478.

Rohlf, J. F. (2016a). TpsDig version 2.26. SUNY at Stony Brook.

Rohlf, J. F. (2016b). TpsUtil. Program version 1.58. SUNY at Stony Brook.

Sheets, H. D. (2016a). CoordGen 8. NY: Canisus College. (Geometric morphometrics software for MacOS). Disponible en: www3.canisius.edu/ sheets/morphsoft. html 
Sheets, H. D. (2016b). MakeFan8, a tool for drawing alignment 'fans' at equal angular spacing. Disponible en: www3.canisius.edu/ sheets/morphsoft.html

Sheets, H. D. (2016c). PCAGen 8. NY: Canisius College. (Geometric morphometrics software for MacOS). Disponible en: www3.canisius.edu/ sheets/morphsoft.html

Smith, A. B., \& Kroh, A. (2011). The echinoid directory.

World wide web electronic publication. http://www. nhm ac.uk/research-curation/projects/echinoid-directory/ index.

Suter, S. J. (1994a). Cladistic analysis of cassiduloid echinoids: trying to see the phylogeny for the trees. Zoological Journal of the Linnean Society, 53, 31-72.
Suter, S. J. (1994b). Cladistic analysis of the living cassiduloids (Echinoidea), and the effects of character ordering and succesive approximations weighting. Zoological Journal of the Linnean Society, 112, 363-387.

Suter, S. J. (1994c). Phylogenetic analyses of cassiduloid echinoids (Echinodermata: Neognathostomata): Morphology, homoplasy and stratigraphic data. Universidad de Chicago, Estados Unidos de América.

Wilkinson, M., Suter, S. J., \& Shires, V. L. (1996). The reduced cladistic consensus method and cassiduloid echinoid phylogeny. Historical biology, 12, 63-73.

Zachos, L. G., \& Molineux, A. (2003). Eocene echinoids of Texas. Journal of paleontology, 77(3), 491-508. 